\title{
Wie weit ist man im vierten Jahr nach dem Examen?
}

Ich finde mich gerade in der Situation wieder, durch meine Klinikstelle in der Kieferorthopädie in Mainz im nächsten Jahr die Fachzahnarztprüfung ablegen zu können. Da rückt der Gedanke über die berufliche Zukunft trotz eines verbleibenden Jahres langsam näher.

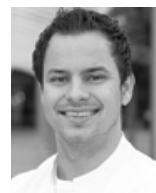

Dr. Philipp F. Gebhardt // Universität Mainz philipp.gebhardt@unimedizin-mainz.de

Dr. Gebhardt berichtet in jeder Ausgabe über seinen Weg vom abgeschlossenen Studium zum

jungen Zahnarzt.

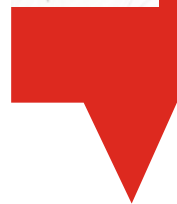

$\mathrm{D}$ urch engen Kontakt zu meinen Studienfreunden bekomme ich gerade bei den allgemeinzahnärztlichen Kollegen mit, dass sich nach einigen Jahren im Angestelltenverhältnis die Frage nach der Selbstständigkeit stellt. Interessant dabei ist, dass die meisten Zusatzqualifikationen im Rahmen eines Curriculums oder Masters erworben haben. Schien dieses Phänomen vor einigen Jahren noch vor allem in Ballungszentren ein Thema gewesen zu sein, ist es inzwischen regionsunabhängig. Gründe für solch eine Spezialisierung liegen oft in der Vertiefung der Fachkenntnisse des bevorzugten Interessenschwerpunktes. Des weiteren bietet sie die Möglichkeit, sich geschickt gegenüber Kollegen positionieren und so bessere Chancen auf dem doch recht hart umkämpften Arbeitsmarkt sichern zu können. Mittlerweile haben nicht nur Kollegen, die sich ernsthaft mit einer Neugründung auseinandersetzen, ein prall gefülltes Portfolio an Fortbildungen und Zertifizierungen. Auch diejenigen, für die schon immer eine Übernahme der elterlichen Praxis feststand, nutzen in der Regel die Möglichkeit, extern Erfahrungen zu sammeln, um so das Behandlungsspektrum zu untermauern oder gar zu erweitern.

Doch welche Möglichkeiten bieten sich überhaupt nach Abschluss der Weiterbildungsassistenz? Da gibt es zum einen den Weg der Weiterführung des Angestelltenverhältnisses zu besseren Konditionen, zum anderen die Selbstständigkeit mit Übernahme oder Neugründung. Die Vorstellung, in der eigenen Praxis keinen Urlaubszettel mehr einreichen zu müssen und auf lange Sicht einen höheren Verdienst zu erzielen, klingt verlockend, birgt jedoch gewisse Risiken. So kommt es oft zu der Überlegung, das Risiko im Rahmen einer Gemeinschaftspraxis mit Kollegen zu teilen. Dabei sollte man sich aber darüber im Klaren sein, dass freundschaftliche Bande bei einer ernsthaften geschäftlichen Zusammenarbeit auf eine harte Probe gestellt werden.

Aus eigenen Erfahrungen kann ich sagen, dass sich Kollegen nach Ende der Assistenzzeit nicht immer direkt in die Selbstständigkeit begeben. Der Hauptgrund dafür ist, weitere fachliche, aber auch betriebswirtschaftliche Erfahrungen sammeln zu können. Doch gerade als vollwertiger Kollege bleibt oft weniger Zeit als vorher, sich mit abrechnungsrelevanten Themen zu beschäftigen. Interessanterweise ist dies selbst für Kollegen mit laufender Praxis unbeliebt und wird gern delegiert. So sollte sich jeder ernsthaft die Frage stellen, ab wann es sinnvoll wird, sich um die Zukunft zu kümmern und ob eine erneute Bindung wirklich Sinn macht. Ratsam ist es in jedem Fall, rechtzeitig anzufangen, sich nach Praxismodellen zu erkundigen und umzuschauen. Denn gerade in Regionen mit hoher Lebensqualität sind attraktive Angebote sehr begehrt und meist schon auf lange Zeit im Voraus vergeben.

\section{Impressum}

Eigentümer \& Copyright @ Springer-Verlag Berlin Heidelberg 2013, Springer Medizin c/o Springer-Verlag GmbH, Tiergartenstr. 17, 69121 Heidelberg, Tel. +49 6221/487-0 Springer Medizin ist ein Teil der Fachverlagsgruppe Springer Science+Business Media Geschäftsführung Springer Medizin: Harm van Maanen (Executive Vice President), Fabian Kaufmann, Dr. Esther Wieland, Matthias Wissel

Director Zahnmedizin: Dr. Nataša Djordjević

Redaktionsleitung "der junge zahnarzt": Susanne Redeker

Redaktion „der junge zahnarzt":

Dr. Swanett Koops (Tel. -8217, swanett.koops@springer.com),

Dr. Stephanie Bissels (Tel. -8138, stephanie.bissels@springer.com)

Layout: Arnulf Illing (Tel. -8952, arnulf.illing@springer.com)

Bildredaktion: Christiane Seufert

Leitung Herstellung: Alison Hepper

Zertifizierte Fortbildung: Dr. Paul Herrmann

Gesamtleitung Sales \& Marketing: Fabian Kaufmann

Anzeigen: Marita Säuberlich (Tel. -8309, Fax -68309, marita.saeuberlich@springer.com) Industriekommunikation: Anja Weisse (Tel. -8739, Fax-68739, anja.weisse@springer.com) Druck: Stürtz GmbH, Würzburg. Printed in Germany

Erscheinungsweise: 4 Ausgaben pro Jahr;

Papierausgabe: ISSN 1869-5744, gedruckt auf säurefreiem Papier

Bezugspreise (unverb. Preisempfehlung inkl. 7\% deutscher MwSt. und Versand): Vor-

zugspreis für persönliche Abonnenten: EUR 49,-; Einzelheftpreis: EUR 20,-. Das Abonnement kann jederzeit 2 Monate vor Ende des Bezugszeitraumes gekündigt werden. Bestellungen oder Rückfragen nimmt jede Buchhandlung oder der Verlag entgegen. Kontakt: Springer Customer Service Center GmbH, Haberstr. 7, 69126 Heidelberg, Tel. +49 6221/345-4303, Fax-4229, Leserservice@springer.com (Mo.-Fr. 8.00 Uhr bis 20.00 Uhr) Copyright \& allgemeine Hinweise: Die Zeitschrift sowie alle in ihr enthaltenen einzelnen Beiträge und Abbildungen sind urheberrechtlich geschützt. Jede Verwertung, die nicht ausdrücklich vom Urheberrechtsgesetz zugelassen ist, bedarf der vorherigen schriftlichen Zustimmung des Verlags. Das gilt insbesondere für Vervielfältigungen, Bearbeitungen, Übersetzungen, Mikroverfilmungen und die Einspeicherung und Verarbeitung in elektronischen Systemen.

Gezeichnete Artikel geben nicht unbedingt die Meinung der Redaktion wieder. Autoren können unter bestimmten Voraussetzungen an der Ausschüttung der Bibliotheks- und Fotokopietantiemen teilnehmen. Einzelheiten bei VG WORT, Abt. Wissenschaft, Goethestr. 49, 80336 München.

Angaben über Dosierungsanweisungen und Applikationsformen sind anhand anderer Literaturstellen oder der Packungsbeilage auf ihre Richtigkeit zu überprüfen. Der Verlag übernimmt keine Gewähr.

Der IVW (Informationsgemeinschaft zur Feststellung der Verbreitung von Werbeträgern) angeschlossen.

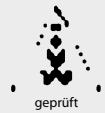

